\title{
Efecto de las descargas del emisario submarino de aguas residuales de la ciudad de Limón sobre la calidad del agua, abundancia y diversidad del fitoplancton en los alrededores de isla Uvita, Costa Rica
}

\section{Effect of the city of Limón marine residual waters outfall discharges on water quality and phytoplankton abundance and diversity in the surroundings of Uvita Island, Costa Rica}

\author{
Nelson Muñoz Simon ${ }^{1 *}$, Lilliana Piedra Castro ${ }^{1}$, Ricardo Jiménez Montealegre ${ }^{1}$, \\ José Pereira Chaves ${ }^{1}$, Meyer Guevara Mora ${ }^{1}$ y Gilberto Piedra Marín ${ }^{2}$
}

\begin{abstract}
RESUMEN
Con el objetivo de evaluar la posible afectación del emisario submarino en la ciudad de Limón, sobre el medio marino circundante, se establecieron 6 estaciones de muestreo, 4 de ellas cercanas a la zona de desfogue y 2 controles; en cada una se tomaron muestras de agua a $0 \mathrm{~m}$ y $10 \mathrm{~m}$ durante el 2012-2013 para medir nutrimentos, parámetros fisicoquímicos, concentración de microalgas y cantidad de unidades formadoras de colonias (UFC) de Enterococcus. Se contrastó la abundancia algal entre las estaciones bajo influencia del emisario, sus controles y entre profundidades, con un análisis de similitud (ANOSIM); en los casos en que hubo diferencia significativa se procedió a realizar un análisis SIMPER para determinar las especies responsables. La abundancia fitoplanctónica se utilizó para jerarquizar la dominancia de especies mediante el diagrama de Olmstead y Tukey e incorporarlas en un análisis de correspondencia canónica. Se determinó que en la zona existe una marcada influencia de las lluvias sobre la concentración de los nutrimentos, silicato y ortofosfato, además de parámetros como el oxígeno disuelto y la salinidad. Las UFC se mantuvieron por debajo del límite máximo de $35 \mathrm{UFC} / 100 \mathrm{~mL}$ establecidos por la Agencia de Protección Ambiental de los Estados Unidos (USEPA). La diversidad fitoplanctónica fue alta y estuvo compuesta, principalmente, por especies cosmopolitas. Debido a que la concentración de nutrientes y UFC son bajas, junto con una alta diversidad algal, se concluye que no existe evidencia directa de un impacto negativo del emisario sobre la zona estudiada.
\end{abstract}

\footnotetext{
1 Escuela de Ciencias Biológicas. Universidad Nacional, Costa Rica. nelson.munoz.simon@una.cr* ORCID: https:// orcid.org/0000-0002-9626-016X, ORCID: https://orcid.org/0000-0003-4878-1531, ORCID: https://orcid.org/00000002-0146-0303, ORCID: https://orcid.org/0000-0001-6056-3364, ORCID https://orcid.org/0000-0002-7873-7489

2 Escuela de Química. Universidad Nacional, Costa Rica. ORCID: https://orcid.org/0000-0003-1332-236X
} 
Palabras clave: Aguas residuales, calidad del agua, Enterococcus, microalgas, nutrientes

\begin{abstract}
In order to assess the possible impact of the marine outfall of the city of Limón on the surrounding marine environment, six sampling stations were established, four near the vent zone and two controls. Water samples were taken in each station $0 \mathrm{~m}$ and $10 \mathrm{~m}$ deep during 20122013 to measure nutrients, physicochemical parameters, microalgae concentration, and colony forming units (CFU) of Enterococcus. Microalgae abundance was compared between stations under outfall influence, their controls and depths using an analysis of similarities (ANOSIM). A SIMPER analysis was also conducted to determine species when significant differences were found. Phytoplankton abundance was also used to rank the dominance of species using the Olmstead-Tukey diagram and a canonical correspondence analysis. Results showed that in the area, there is a marked influence of rainfall on nutrients, silicate and orthophosphate, in addition to parameters such as dissolved oxygen and salinity. The dissolved oxygen concentration was high, reflecting that there is no significant organic load in the environment, while CFUs remained in ranges well below the maximum limits $(35 \mathrm{UFC} / 100 \mathrm{~mL})$ set by the United States Environmental Protection Agency (USEPA). Phytoplankton diversity was high and composed mostly of cosmopolitan species. Due to the low nutrient concentration and CFU counts, together with the high microalgae diversity, it is concluded that there is no direct evidence of a negative impact of the marine outfall on the studied area.
\end{abstract}

Keywords: Wastewater, water quality, Enterococcus, microalgae, nutrients

\section{INTRODUCCIÓN}

Un emisario submarino es considerado la etapa final de todo un proceso de tratamiento de aguas residuales. Está conformado por varios difusores que se ubican en el fondo de la columna de agua y hacia los cuales se bombean las aguas tratadas; la descarga forma una pluma que se desplaza por debajo de la termoclina, al mismo tiempo que se diluye, y evita que los efluentes cargados de nutrientes y patógenos lleguen a la superficie donde podrían causar problemas de contaminación (Parnell, 2003; Ramírez \& Salazar, 2005).
Países como Estados Unidos (Parnell, 2003), Chile (Toledo et al. 2005), Argentina (Cuello et al. 2019), y algunos otros han empleado emisarios submarinos desde hace muchos años con resultados prometedores, por ello este tipo de estructura se ha vuelto muy común en el mundo, incluso en Latinoamérica, donde actualmente se encuentran en operación unas 104 unidades (Salas, 2000).

En el año 2005 iniciaron las operaciones del primer emisario submarino de Costa Rica, ubicado en la ciudad de Limón, el cual beneficia a unos 70000 habitantes (Carvajal, 2005). La estructura es administrada por una institución autónoma del Estado 
Efecto de las descargas del emisario submarino de aguas residuales de la ciudad de Limón sobre la calidad del agua, abundancia y diversidad del fitoplancton en los alrededores de isla Uvita, Costa Rica

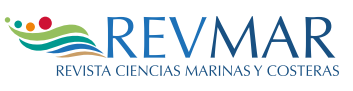

costarricense (Instituto Costarricense de Acueductos y Alcantarillados), tiene una capacidad de flujo de $200 \mathrm{l} / \mathrm{s}$, y su tubería se adentra unos $800 \mathrm{~m}$ en el mar, donde se hace la liberación de los efluentes a una profundidad de $17 \mathrm{~m}$ (Ávalos, 2004).

Este tipo de proyecto ha generado cuestionamientos sobre la posible afectación en el medio circundante, pues es necesario garantizar a la población el mínimo impacto en el ecosistema marino; por ello el Estado costarricense se ha comprometido, a través del ente operador, a mantenerse vigilante de la correcta operación del emisario mediante un programa de monitoreo (PROIGE- AyA, 1999). Estos programas permiten dar un seguimiento continuo para evaluar la correcta operación, y, en caso de anomalías, dar la alerta temprana para ejecutar las medidas correctivas.

Es necesario garantizar que los parámetros a vigilar sean los más adecuados para la toma de decisiones (Gianesella et al. 1999). Debido a que uno de los efectos más perjudiciales de este tipo de obra está relacionados con la eutrofización (Parnell, 2003), es necesario llevar a cabo un programa de muestreo que sea capaz de discernir cuál o cuáles parámetros fisicoquími$\cos$ y biológicos podrían haber sido alterados de manera activa por las descargas del emisario; o en su defecto, brindar indicadores que, de manera rápida y económicamente rentable, sean capaces de brindar información sobre el estado del ecosistema.

El presente estudio tuvo como finalidad evaluar la calidad del agua, la abundancia y diversidad fitoplanctónica en 6 puntos alrededor de isla Uvita, para establecer si existen efectos negativos causados por las descargas del emisario submarino de la ciudad de Limón sobre el medio circundante.

\section{MATERIALES Y MÉTODOS}

La zona de estudio se ubica en el Caribe costarricense. El promedio de lluvias es de $3574.9 \mathrm{~mm}$ y no se presenta una estación seca bien definida; en septiembre la precipitación promedio alcanza $45.3 \mathrm{~mm}$, lo que corresponde al valor mensual mínimo (Instituto Meteorológico Nacional, 2016). Se reconocen 2 periodos bien definidos de lluvia: de noviembre a enero, y de mayo a agosto (Instituto Meteorológico Nacional, 2016), siendo diciembre y julio los meses más lluviosos, con promedios de precipitación de $440.6 \mathrm{~mm}$ y $438.2 \mathrm{~mm}$ respectivamente. De acuerdo con el registro histórico el año con mayores precipitaciones fue 1944 y el más seco 1995 (Instituto Meteorológico Nacional, 2016). Las temperaturas más bajas se registran entre diciembre y enero, y las más altas durante los meses de mayo a septiembre (Instituto Meteorológico Nacional, 2016, Marín et al. 2009). 
El emisario submarino se encuentra ubicado a 800 metros de la costa, a una profundidad de $20 \mathrm{~m}$, está conformado por un tramo de $200 \mathrm{~m}$, dotado de 30 difusores y una capacidad de desfogue de $200 \mathrm{~L} / \mathrm{s}$. Limita al este con la isla Uvita y al oeste con la costa de Limón. Entre ambos puntos se define un pasaje natural con una corriente marina que se dirige de norte a sur, a una velocidad promedio de 0.90 km/h (PROIGE-AyA, 1999).

Los valores de precipitación de la zona fueron obtenidos de: Morera (2012), Naranjo \& Poleo (2012); Naranjo (2013) y Chinchilla (2013).

En total se llevaron a cabo 4 muestreos para registrar los parámetros físico-químicos y biológicos, uno por mes, entre setiembre y octubre del 2012, y marzo y setiembre del 2013, en 6 estaciones en los alrededores de isla Uvita (Fig. 1). Las estaciones E1 (ubicada sobre el emisario), E2, E3 y E4 se designaron como zonas bajo influencia, mientras que E5 y E6 se utilizaron como puntos de control. En todas las estaciones se tomaron 2 muestras de 3 L con una botella Niskin, una a $0 \mathrm{~m}$ y otra a $10 \mathrm{~m}$ de profundidad. Del volumen total se apartó una alícuota de 250 $\mathrm{ml}$ para medir in situ la concentración de oxígeno disuelto, la salinidad y la temperatura con un multiparámetro HANNA, modelo HI9033. Mientras que el pH se midió con un potenciómetro HANNA, modelo HI98130.

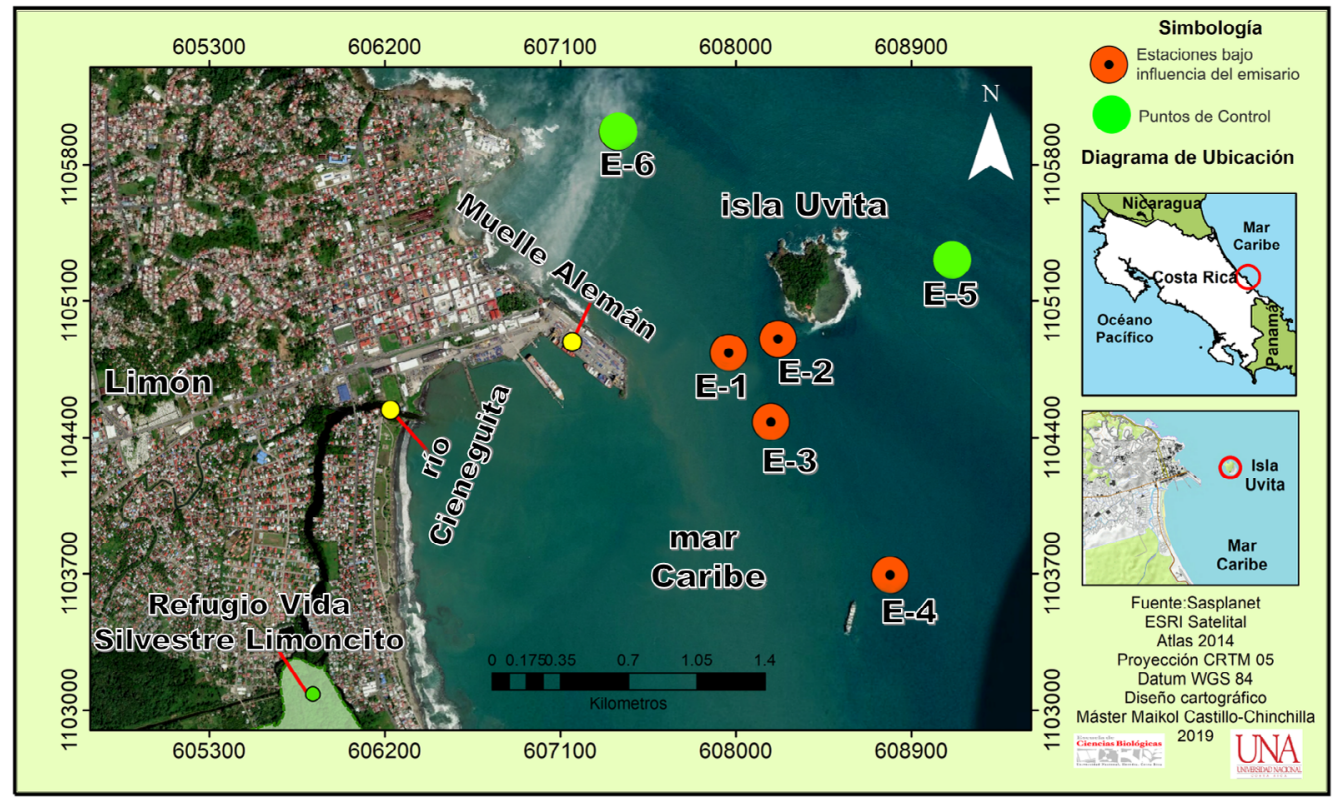

Fig. 1. Área de estudio y ubicación estaciones de muestreo

Fig. 1. Study area and location of sampling stations 
Efecto de las descargas del emisario submarino de aguas residuales de la ciudad de Limón sobre la calidad del agua, abundancia y diversidad del fitoplancton en los alrededores de isla Uvita, Costa Rica

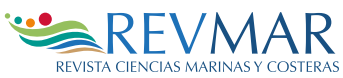

Del volumen restante se tomaron $250 \mathrm{ml}$ para medir sólidos totales y 250 $\mathrm{ml}$ para cada uno de los nutrientes (nitrato, nitrito, amonio-amoniaco, ortofosfatos y silicatos). Todas las muestras se mantuvieron en hielo hasta su llegada al laboratorio de Química Marina de la Universidad Nacional en Heredia, donde fueron conservadas a $-10^{\circ} \mathrm{C}$ para su posterior análisis. Para la determinación de amonio-amoniaco, las muestras se fijaron previamente con unas gotas de ácido sulfúrico concentrado.

Los sólidos totales se midieron con la metodología propuesta por Clesceri et al. (1999). Las tres formas inorgánicas del nitrógeno disuelto se determinaron de la siguiente forma: $\mathrm{El}$ amonio-amoniaco $\left(\mathrm{N}-\mathrm{NH}_{4}^{+}+\mathrm{N}-\mathrm{NH}_{3}\right)$ se cuantificó según el método del azul de indofenol (Tinoco, 2007), el nitrato $\left(\mathrm{N}-\mathrm{NO}_{3}^{-}\right)$mediante la espectrofotometría ultravioleta (Hansen y Koroleff, 2007), mientras que el nitrito $\left(\mathrm{N}_{-} \mathrm{NO}_{2}{ }^{-}\right)$ según la metodología 420 de Hansen y Koroleff (2007). Por su parte, la concentración de ortofosfato inorgánico disuelto $\left(\mathrm{P}_{-} \mathrm{PO}_{4}^{-3}\right)$ se determinó con el método colorimétrico del ácido vanado-molibdo-fosfórico (Hansen \& Koroleff, 2007), y los silicatos se analizaron por el método del metol, según lo propone Tinoco (2007).

Para determinar la densidad algal se tomaron submuestras de un litro y se fijaron con Lugol ácido. Después se procesaron según el método de concentración en probetas, y la cantidad de algas en cada estación se contó por triplicado, en celdas de Palmer-Maloney (Sournia, 1978).

Adicionalmente, se tomaron muestras cualitativas de fitoplancton en cada estación, haciendo arrastres horizontales de 2 minutos con una red de fitoplancton de 20 micrómetros. El volumen final de cada colecta se fijó con Lugol ácido y se mantuvo en la oscuridad. Para cada muestra se identificaron las microalgas presentes en el nivel de especie, con la ayuda de un microscopio de luz Olympus, modelo BX 75. Los datos obtenidos en los conteos se utilizaron para calcular los índices de diversidad de Shannon y de dominancia de Simpson, los cuales se promediaron tanto para las estaciones con influencia como para los controles. También, los datos se utilizaron para calcular las frecuencias de aparición de cada especie.

La cuantificación de Unidades Formadoras de Colonias (UFC) de Enterococcus se llevó a cabo según el método 1600 propuesto por la USEPA (2006), a partir de muestras de $300 \mathrm{ml}$ tomadas en cada estación de muestreo y dos controles positivos ubicados bajo el puente del río Cieneguita y su desembocadura.

Los datos físico-químicos y ambientales se integraron en un análisis de componentes principales (Varona-Cordero et al. 2010) que utilizan la extensión XLSTAT (versión 2018) para MS Excel. A continuación, se 
realizó un PERMANOVA para establecer diferencias estadísticamente significativas en la matriz ambiental entre fondo vs superficie y zona de influencia vs controles, previa estandarización de los datos $(P<0.05)$, por medio del paquete Vegan en R (Oksanen et al. 2019).

Los datos biológicos transformados a la cuarta raíz y normalizados se utilizaron para llevar a cabo un análisis ANOSIM y SIMPER (Vidal et al. 2017; Stanca \& Parsons, 2017) que sigue los mismos criterios establecidos para los factores físico-químicos. Por otra parte, la dominancia de especies fue jerarquizada mediante el diagrama de Olmstead-Tukey (Sokal \& Rohlf, 1970). Finalmente, las especies dominantes obtenidas con el diagrama de Olmstead-Tukey se utilizaron para llevar a cabo un Análisis de Correspondencia Canónica (Varona-Cordero et al. 2010).

\section{RESULTADOS}

Los valores de precipitación pluvial para noviembre y octubre del 2012 fueron de $139.6 \mathrm{~mm}$ y $88 \mathrm{~mm}$ respectivamente, mientras que para marzo y setiembre de 2013 fueron de $315.5 \mathrm{~mm}$ y $111.9 \mathrm{~mm}$ correspondientemente.

En el análisis de componentes principales (ACP, Fig. 2) los 3 primeros ejes explican el $87.3 \%$ del total de la variabilidad. Las variables precipitación (0.89), silicatos $(0.35)$ y oxígeno disuelto (0.27) aportaron la mayor contribución en el primer componente (PC1). En el segundo componente (PC2) fueron las variables silicatos (0.88), amonio (0.61) y temperatura.

En el gráfico biplot, entre el primer y cuarto cuadrante se ubican aquellas estaciones de muestreo que se distribuyen a lo largo de un gradiente de concentración de silicato, nitrato y ortofosfato, la gran mayoría de ellas corresponden al primer muestreo y solamente 3 al cuarto (Fig. 2). Las mayores concentraciones de ortofosfatos y nitrato se registraron durante el primer muestreo, mientras que los valores de silicatos fueron mayores en el tercero y el primero.

En los cuadrantes I y II se ubican las estaciones cuyos datos fueron obtenidos en el tercer muestreo, en ellas existe una clara separación entre la superficie y el fondo (designados como A y B comparativamente). La variación en $\mathrm{A}$, donde se ubican estaciones de superficie, está claramente definida por el silicato, el oxígeno disuelto y la precipitación, mientras que en $\mathrm{B}$ hay una influencia de las precipitaciones y el oxígeno disuelto. En el tercer cuadrante se ubicaron los puntos de colecta relacionados con una disminución de la precipitación, los silicatos y un leve aumento del ortofosfato y el nitrato, sobre todo durante el segundo muestreo. 
Efecto de las descargas del emisario submarino de aguas residuales de la ciudad de Limón sobre la calidad del agua, abundancia y diversidad del fitoplancton en los alrededores de isla Uvita, Costa Rica

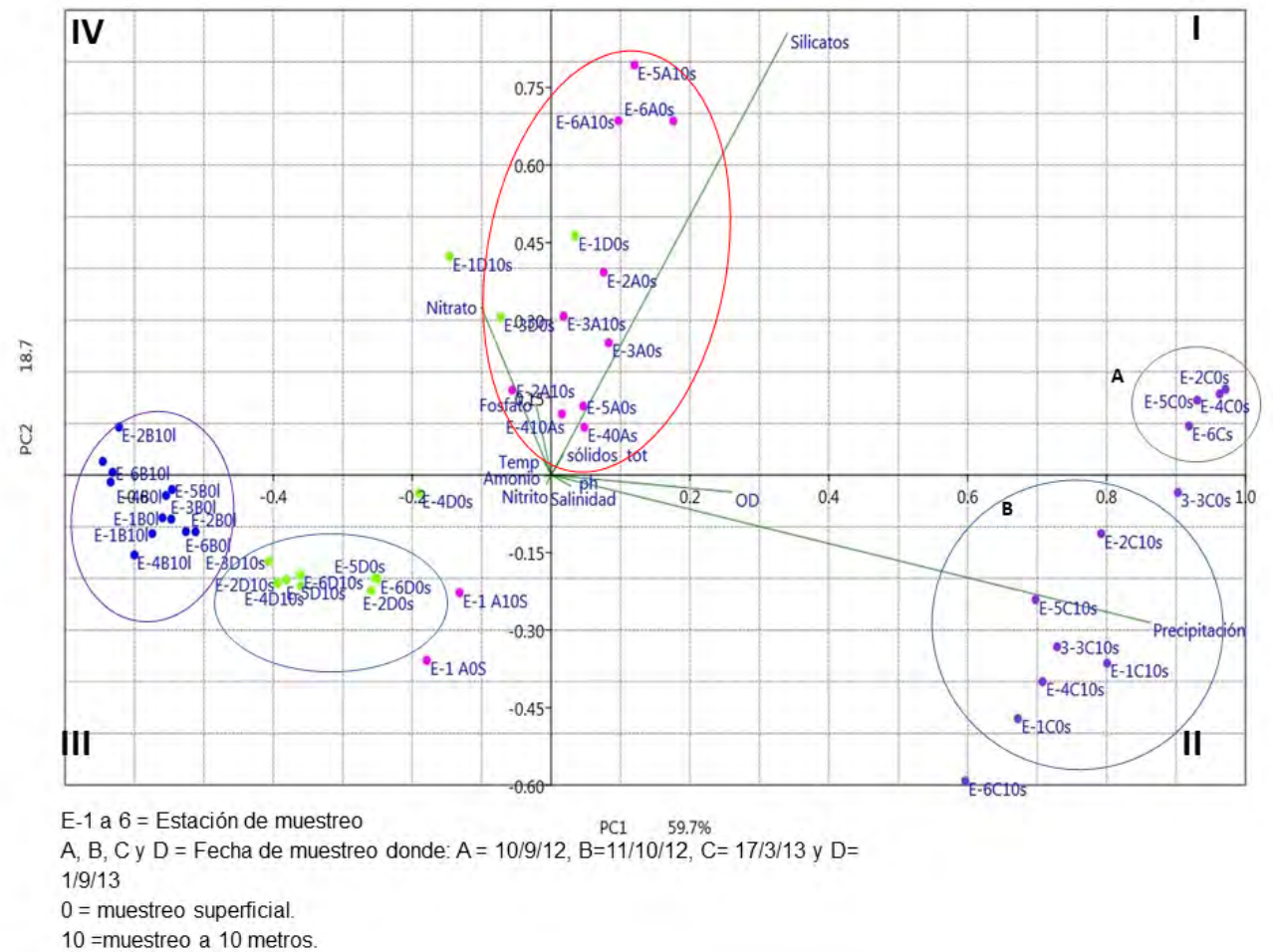

Fig. 2. Biplot del análisis de componentes principales (ACP) de las variables fisicoquímicas en estaciones bajo influencia del emisario submarino de Limón y sus controles según profundidad

Fig. 2. Principal Components Biplot of the physicochemical variables in stations under the influence of Limón's marine outfall and its controls according to depth

Según los resultados del PER- control positivo, con valores de 1800 MANOVA, las variables fisicoquímicas arrojaron leves diferencias entre la superficie y el fondo $\left(\mathrm{R}^{2}=0.08047\right.$, $\mathrm{F}(1 ; 47)=4.0257 ; P<0.05)$, mientras que los controles y las zonas de influencia mostraron ser similares entre ellos $\left(\mathrm{R}^{2}=0.00743\right.$, F $(1 ; 47)=$ $0.34421, P>0.05)$.

La mayor cantidad de unidades formadoras de colonias de Enterococcus se obtuvo en los puntos de y 1550 UFC en las estaciones puente del río Cieneguita y desembocadura, respectivamente (Cuadro 1). En los puntos con influencia del emisario submarino la mayor cantidad de UFC se registró en la estación E-1, con un total de 19.5 UFC durante octubre del 2012. En las estaciones control, el mayor conteo se obtuvo en la estación E- 6 con un total de 12 UFC y el menor de 1 UFC en E-5. 
Cuadro 1. Cantidad de Unidades Formadoras de Colonias de Enterococcus dentro y fuera de la zona influencia del emisario submarino de Limón durante el período 2012-2013

Table 1. Amount of Enterococcus CFUs inside and outside the influence zone of Limón's marine outfall during the 2012-2013 period

\begin{tabular}{lccccccccc}
\hline & & & \multicolumn{2}{c}{$\begin{array}{c}\text { Estaciones bajo influencia } \\
\text { del emisario }\end{array}$} & \multicolumn{2}{c}{$\begin{array}{l}\text { Estaciones } \\
\text { sin influencia } \\
\text { del emisario }\end{array}$} \\
\hline $\begin{array}{l}\text { Fecha de } \\
\text { muestreo }\end{array}$ & $\begin{array}{l}\text { Puente } \\
\text { Cieneguita }\end{array}$ & $\begin{array}{l}\text { Desembo- } \\
\text { cadura rín } \\
\text { Cieneguita }\end{array}$ & E-1 & E-2 & E-3 & E-4 & E-5 & E-6 \\
$10 / 9 / 2012$ & N/A & N/A & N/A & N/A & N/A & N/A & N/A & N/A \\
$11 / 10 / 2012$ & 1800 & 1550 & 19.5 & 16.5 & 4 & 5 & 12 & 17 \\
$17 / 3 / 2013$ & 900 & 850 & 1 & 2.5 & 2.5 & 4 & 1 & 2.5 \\
$1 / 9 / 2013$ & 524 & 395 & 4 & 19 & 3.5 & 8 & 2 & 2 \\
\hline
\end{tabular}

A partir del análisis de varianza de una vía, no se determinó diferencia significativa en relación con la cantidad de unidades formadoras de colonias de Enterococcus entre las zonas bajo influencia del emisario submarino y las zonas fuera de su influencia $(P=0.99$, $\alpha=0.005$, gl 23). Al comparar las zonas con influencia contra los controles positivos (puente y desembocadura del río Cieneguita), se obtuvieron diferencias significativas $(P=0.004, \alpha=$ $0.005, \mathrm{gl} 23$ y $P=0.002, \alpha=0.005, \mathrm{gl}$ 23 , en orden).

En las estaciones bajo influencia del emisario la mayor densidad algal fue de 28100 cél/L, misma que correspondió a la E-2 durante marzo de 2013 (Fig. 3), y la densidad más baja registrada fue de 500 cél/L en la estación 4 , en octubre de 2012.
A 10 metros de profundidad las estaciones ubicadas cerca de la zona de desfogue obtuvieron un máximo de 16200 cél/L en la estación E-3, en marzo de 2013 y un mínimo de 5150 cél/L en E-4, durante marzo del mismo año. El segundo valor en importancia se registró en E-2 con un total de 12 550 cél/L, en setiembre del 2012.

Las estaciones sin influencia del emisario, ubicadas a la profundidad de 10 metros, obtuvieron un promedio de 5500 cél/L, siendo la E-6 la que reporta la concentración más alta de fitoplancton (8 700 cél/L), mientras que el valor mínimo de 2600 cél/L se reportó en la E-5, en marzo de 2013.

En las muestras de red (Cuadro 2) se identificaron 123 especies microalgales, de ellas 86 fueron diatomeas, 29 dinoflagelados, 5 cianobacterias y 3 
Efecto de las descargas del emisario submarino de aguas residuales de la ciudad de Limón sobre la calidad del agua, abundancia y diversidad del fitoplancton en los alrededores de isla Uvita, Costa Rica
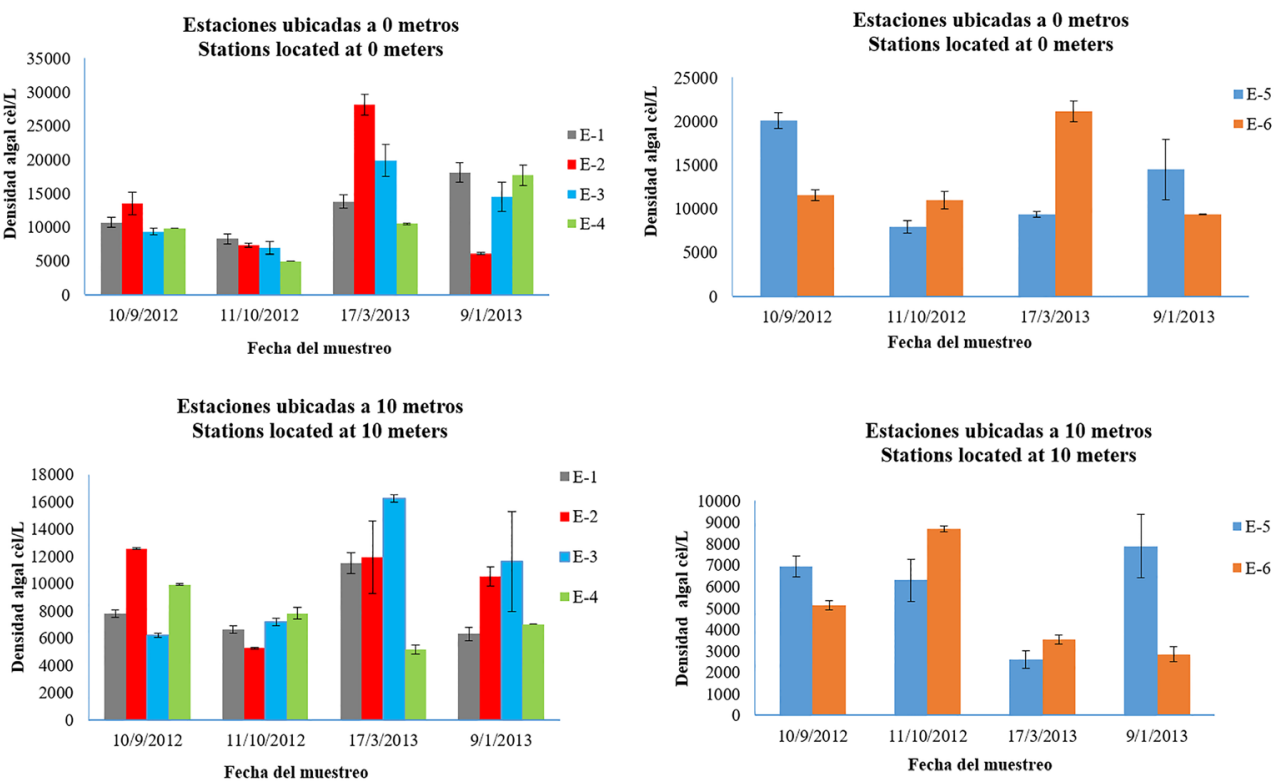

Fig. 3. Concentración de microalgas a 0 y 10 metros en 4 estaciones bajo la influencia del emisario submarino de Limón y sus controles

Fig. 3. Microalgae concentration 0 and 10 meters deep in four stations within the influence of Limón's marine outfall and its controls

corresponden a fitoflagelados diversos. Dentro de las diatomeas Skeletonema costatum, Rhizosolenia stolterfothii, Thalassionema nitzschioides y varias especies de Chaetoceros sp. son muy frecuentes, tanto espacial como temporalmente. Dentro de las especies menos comunes que se identificaron se pueden citar: Odontella longicruris, Oltmannsiellopsis sp. y algunas diatomeas pennadas que solo se observaron en uno o dos muestreos.
La diversidad promedio en la zona bajo influencia del emisario submarino en el nivel de superficie mostró poca variación entre las fechas de muestreo (Fig. 4). Los valores oscilaron entre 2.30 bits/cél a 2.87 bits/ cél, mientras que el promedio de la dominancia se mostró baja. En las estaciones control, el índice de Shannon registró valores más altos en octubre del 2012 y el 17 de marzo de 2013, que coinciden con una disminución de la dominancia. 
Cuadro 2. Especies fitoplanctónicas presentes en 4 estaciones bajo influencia del emisario submarino de Limón y 2 fuera de esa influencia

Table 2. Phytoplankton species present in four stations under the influence of Limón's marine outfall and two without influence

\section{Estación}

\begin{tabular}{|c|c|c|c|c|c|c|}
\hline Especie & E-1 & E-2 & E-3 & E-4 & E-5 & E-6 \\
\hline \multicolumn{7}{|l|}{ Bacillariophyceae } \\
\hline $\begin{array}{l}\text { Actinoptychus undulatus } \\
\text { (Kützing) Ralfs } 1861\end{array}$ & + & & + & & + & \\
\hline Amphiprora sp. & + & & + & & & \\
\hline Asterionella japonica Cleve 1882 & +++ & ++ & ++ & ++ & + & ++ \\
\hline $\begin{array}{l}\text { Asteromphalus hyalinus Karstsen } \\
1905\end{array}$ & ++ & + & & & + & +++ \\
\hline $\begin{array}{l}\text { Bacteriastrum delicatulum Cleve } \\
1897\end{array}$ & +++ & +++ & +++ & +++ & ++ & ++ \\
\hline $\begin{array}{l}\text { Bacteriastrum elongatum Cleve } \\
1897\end{array}$ & +++ & ++ & +++ & +++ & +++ & + \\
\hline $\begin{array}{l}\text { Biddulphia mobiliensis Grunow } \\
1882\end{array}$ & & & + & ++ & + & + \\
\hline $\begin{array}{l}\text { Ceratoneis closterium Ehrenberg } \\
1839\end{array}$ & & & + & + & & + \\
\hline $\begin{array}{l}\text { Cerataulina pelagica Hendey } \\
1937\end{array}$ & ++ & +++ & + & ++ & ++ & +++ \\
\hline Chaetoceros affinis Lauder 1864 & ++++ & ++++ & +++ & ++ & ++++ & +++ \\
\hline $\begin{array}{l}\text { Chaetoceros atlanticus Cleve } \\
1873\end{array}$ & ++++ & +++ & +++ & ++++ & +++ & +++ \\
\hline Chaetoceros brevis F.Schütt 1895 & + & + & & + & & \\
\hline $\begin{array}{l}\text { Chaetoceros cf. mitra (Bailey) } \\
\text { Cleve } 1896\end{array}$ & + & & + & ++ & ++ & + \\
\hline $\begin{array}{l}\text { Chaetoceros coarctatus Lauder } \\
1864\end{array}$ & + & ++ & & & + & + \\
\hline $\begin{array}{l}\text { Chaetoceros comprsessus Lauder } \\
1864\end{array}$ & ++ & + & + & ++ & ++ & \\
\hline $\begin{array}{l}\text { Chaetoceros constrictus Gran } \\
1897\end{array}$ & +++ & ++ & + & & + & ++ \\
\hline $\begin{array}{l}\text { Chaetoceros costatus Pavillard } \\
1911\end{array}$ & + & + & + & + & + & + \\
\hline $\begin{array}{l}\text { Chaetoceros curvisetus Cleve } \\
1889\end{array}$ & ++++ & ++++ & +++ & +++ & ++++ & + \\
\hline
\end{tabular}


Efecto de las descargas del emisario submarino de aguas residuales de la ciudad de Limón sobre la calidad del agua, abundancia y diversidad del fitoplancton en los alrededores de isla Uvita, Costa Rica

\begin{tabular}{|c|c|c|c|c|c|c|}
\hline \multicolumn{7}{|l|}{ Estación } \\
\hline Especie & E-1 & E-2 & E-3 & E-4 & E-5 & E-6 \\
\hline $\begin{array}{l}\text { Chaetoceros decipiens Cleve } \\
1873\end{array}$ & ++++ & ++++ & $\begin{array}{l}++ \\
++\end{array}$ & ++++ & +++ & +++ \\
\hline $\begin{array}{l}\text { Chaetoceros didymus Ehrenberg } \\
1845\end{array}$ & +++ & & +++ & ++ & ++ & ++ \\
\hline Chaetoceros diversus Cleve 1873 & + & + & + & & & + \\
\hline $\begin{array}{l}\text { Chaetoceros laciniosus Schütt } \\
1895\end{array}$ & +++ & ++ & + & ++++ & + & +++ \\
\hline $\begin{array}{l}\text { Chaetoceros laevis Leuduger- } \\
\text { Fortmorel } 1892\end{array}$ & +++ & +++ & ++ & +++ & +++ & ++ \\
\hline $\begin{array}{l}\text { Chaetoceros lorenzianus Grunow } \\
1863\end{array}$ & + & + & + & + & & + \\
\hline $\begin{array}{l}\text { Chaetoceros pendulus Karsten } \\
1905\end{array}$ & + & & & + & & \\
\hline $\begin{array}{l}\text { Chaetoceros peruvianus } \\
\text { Brightwell } 1856\end{array}$ & +++ & + & ++ & +++ & & ++ \\
\hline $\begin{array}{l}\text { Chaetoceros pseudocurvisetus } \\
\text { Mangin } 1910\end{array}$ & + & & + & + & + & \\
\hline Chaetoceros sp1. & ++ & + & & + & & \\
\hline Chaetoceros sp2. & ++ & + & & + & + & + \\
\hline Chaetoceros sp3. & & & & & + & \\
\hline Chaetoceros subtilis Cleve 1896 & ++ & + & ++ & + & ++ & ++ \\
\hline $\begin{array}{l}\text { Cylindrotheca closterium } \\
\text { (Ehrenberg) Reimann \& J. C. } \\
\text { Lewin, } 1964\end{array}$ & + & & ++ & ++ & ++ & + \\
\hline Corethron sp. & + & + & & & & ++ \\
\hline $\begin{array}{l}\text { Coscinodiscus concinnus W. } \\
\text { Smith } 1856\end{array}$ & +++ & ++ & ++ & +++ & +++ & +++ \\
\hline $\begin{array}{l}\text { Cyclotella comensis } \\
\text { Grunow } 1882\end{array}$ & & + & & + & + & + \\
\hline $\begin{array}{l}\text { Cyclotella meneghiniana } \\
\text { Kützing } 1844\end{array}$ & ++ & + & + & + & + & + \\
\hline Cyclotella sp. & ++ & ++ & + & + & + & + \\
\hline Cymbella sp. & & ++ & & + & & \\
\hline $\begin{array}{l}\text { Dactyliosolen } \\
\text { fragilissimus (Bergon) Hasle } \\
1996\end{array}$ & & + & + & + & + & + \\
\hline
\end{tabular}




\begin{tabular}{|c|c|c|c|c|c|c|}
\hline \multicolumn{7}{|l|}{ Estación } \\
\hline Especie & E-1 & E-2 & E-3 & E-4 & E-5 & E-6 \\
\hline Dactyliosolen $\mathrm{sp}$. & + & & & & & \\
\hline $\begin{array}{l}\text { Ditylum brightwellii (T. West) } \\
1885\end{array}$ & ++ & + & ++ & ++ & ++ & + \\
\hline $\begin{array}{l}\text { Eucampia cf zodiacus Ehrenberg } \\
1839\end{array}$ & & + & + & & + & + \\
\hline Grammatophora sp. & +++ & + & + & & & \\
\hline $\begin{array}{l}\text { Guinardia striata (Stolterfoth) } \\
\text { Hasle } 1996\end{array}$ & ++ & + & & +++ & ++++ & $\begin{array}{l}++ \\
++\end{array}$ \\
\hline $\begin{array}{l}\text { Guinardia flaccida (Castracane) } \\
\text { H. Peragallo } 1892\end{array}$ & +++ & ++ & ++ & & ++ & ++ \\
\hline $\begin{array}{l}\text { Hemiaulus hauckii Grunow ex } \\
\text { Van Heurck } 1882\end{array}$ & +++ & +++ & +++ & ++ & +++ & +++ \\
\hline $\begin{array}{l}\text { Hemiaulus membranaceus Cleve } \\
1873\end{array}$ & ++++ & +++ & ++ & +++ & ++++ & +++ \\
\hline Lauderia annulata Cleve 1873 & & & + & + & & \\
\hline $\begin{array}{l}\text { Leptocylindrus danicus Cleve } \\
1889\end{array}$ & ++++ & +++ & +++ & +++ & ++++ & $\begin{array}{l}++ \\
++\end{array}$ \\
\hline $\begin{array}{l}\text { Lioloma pacificum (Cupp) Hasle } \\
1996\end{array}$ & ++ & + & & & & + \\
\hline $\begin{array}{l}\text { Lithodesmium undulatum } \\
\text { Ehrenberg } 1839\end{array}$ & + & & & & + & + \\
\hline Melosira sp. & & + & + & & & + \\
\hline $\begin{array}{l}\text { Meuniera membranacea (Cleve) } \\
\text { P.C.Silva } 1996\end{array}$ & + & + & + & & + & \\
\hline Navicula sp. & + & + & + & + & ++ & ++ \\
\hline $\begin{array}{l}\text { Nitzschia pungens Grunow ex } \\
\text { Cleve } 1897\end{array}$ & ++ & + & + & + & + & + \\
\hline $\begin{array}{l}\text { Nitzschia pungens var atlantica } \\
\text { Cleve } 1897\end{array}$ & +++ & ++ & ++ & ++ & ++ & ++ \\
\hline $\begin{array}{l}\text { Odontella longicruris (Greville) } \\
\text { M.A. Hoban } 1983\end{array}$ & + & & & & & \\
\hline Oltmannsiellopsis Sp. & & & + & & & + \\
\hline Pinnularia sp. & + & & + & + & & \\
\hline $\begin{array}{l}\text { Pleurosigma angulatum (J. T. } \\
\text { Quekett) W. Smith } 1852\end{array}$ & + & + & ++ & + & & + \\
\hline Pleurosigma sp. & + & & + & & & \\
\hline
\end{tabular}


Efecto de las descargas del emisario submarino de aguas residuales de la ciudad de Limón sobre la calidad del agua, abundancia y diversidad del fitoplancton en los alrededores de isla Uvita, Costa Rica

\section{Estación}

\begin{tabular}{|c|c|c|c|c|c|c|}
\hline Especie & E-1 & E-2 & E-3 & E-4 & E-5 & E-6 \\
\hline $\begin{array}{l}\text { Proboscia alata (Brightwell) } \\
\text { Sundström, } 1986\end{array}$ & + & + & +++ & ++ & ++ & ++ \\
\hline Pseudo-nitszchia sp. & + & + & & & & \\
\hline $\begin{array}{l}\text { Pseudo-nitzschia pungens } \\
\text { (Grunow ex Cleve) Hasle } 1993\end{array}$ & ++ & ++ & ++ & ++ & +++ & +++ \\
\hline $\begin{array}{l}\text { Pseudosolenia calcar-avis } \\
\text { (Schultze) B. G. Sundström } 1986\end{array}$ & +++ & ++ & ++ & + & + & ++ \\
\hline $\begin{array}{l}\text { Rhizosolenia acuminata } \\
\text { (H.Peragallo) H. Peragallo } 1907\end{array}$ & + & & + & & & \\
\hline $\begin{array}{l}\text { Rhizosolenia hebetata Bailey } \\
1856\end{array}$ & & & + & & + & ++ \\
\hline $\begin{array}{l}\text { Rhizosolenia robusta } \text { G. Norman } \\
\text { ex Ralfs } 1861\end{array}$ & & + & + & & ++ & + \\
\hline $\begin{array}{l}\text { Rhizosolenia } \text { cf. polydactyla } \\
\text { Castracane } 1886\end{array}$ & + & & & + & & \\
\hline $\begin{array}{l}\text { Rhizosolenia imbricata } \\
\text { Brightwell } 1858\end{array}$ & + & & & & + & + \\
\hline $\begin{array}{l}\text { Rhizosolenia setigera Brightwell } \\
1858\end{array}$ & +++ & + & +++ & & ++++ & ++ \\
\hline $\begin{array}{l}\text { Rhizosolenia stolterfothii } \mathrm{H} . \\
\text { Peragallo } 1888\end{array}$ & ++++ & ++++ & $\begin{array}{l}++ \\
++\end{array}$ & ++++ & ++++ & $\begin{array}{l}++ \\
++\end{array}$ \\
\hline $\begin{array}{l}\text { Skeletonema costatum (Greville) } \\
\text { Cleve } 1878\end{array}$ & ++++ & ++++ & $\begin{array}{l}++ \\
++\end{array}$ & ++++ & ++++ & $\begin{array}{l}++ \\
++\end{array}$ \\
\hline Stephanopyxis sp. & + & + & & + & & \\
\hline $\begin{array}{l}\text { Thalassionema nitzschioides } \\
\text { (Grunow) Mereschkowsky } 1902\end{array}$ & ++++ & ++++ & $\begin{array}{l}++ \\
++\end{array}$ & ++++ & ++++ & $\begin{array}{l}++ \\
++\end{array}$ \\
\hline $\begin{array}{l}\text { Thalassiosira nana Lohmann } \\
1908\end{array}$ & + & + & & & + & + \\
\hline Thalassiosira sp. & & & ++ & +++ & ++ & + \\
\hline $\begin{array}{l}\text { Thalassiothrix frauenfeldii } \\
\text { Grunow } 1880\end{array}$ & & ++ & ++ & ++ & ++ & + \\
\hline $\begin{array}{l}\text { Thalassiothrix longissima Cleve } \\
\text { \& Grunow } 1880\end{array}$ & + & ++ & + & ++ & ++ & ++ \\
\hline \multicolumn{7}{|l|}{ Dinophyceae } \\
\hline $\begin{array}{l}\text { Ceratium furca (Ehrenberg) } \\
\text { Claparède \& Lachmann } 1859\end{array}$ & ++++ & +++ & $\begin{array}{l}++ \\
++\end{array}$ & ++ & & $\begin{array}{l}++ \\
++\end{array}$ \\
\hline
\end{tabular}




\section{Estación}

\begin{tabular}{l}
\hline Especie \\
\hline Ceratium fusus (Ehrenberg) \\
Dujardin 1841 \\
Ceratium horridum (Cleve) Gran \\
1902
\end{tabular}

Ceratium macroceros

(Ehrenberg) Vanhöffen 1897

Ceratium pentagonum Gourret + 1883

Ceratium sp.

Ceratium trichocereus

(Ehrenberg) Kofoid 1908

Ceratium tripos (O.F.Müller)

Nitzsch 1817

Dinophysis caudata Saville-Kent 1881

Gonyaulax digitale (Pouchet)

Kofoid 1911

Gymnodinium sp.

Gymnodinium sp. redondeado

Gymnodinium cf. pulchellum J.

Larsen 1994

Gyrodinium sp.

Heterocapsa $\mathrm{sp}$.

Phalacroma rotundatum

(Claparéde \& Lachmann) Kofoid

E-1

E-2

E-3

E-4

E-5 E-6

$+$

$+$

$+\quad+\quad+$

$++$

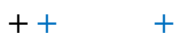

$++$

$+$

$+$

$\begin{array}{lll}+ & + \\ + & +\end{array}$

\& J. R. Michener 1911

Prorocentrum micans Ehrenberg 1834

Prorocentrum mínimum

(Pavillard) J.Schiller 1933

Protoperidinium conicum (Gran)

Balech 1974

Protoperidinium divergens

(Ehrenberg) Balech 1974

Protoperidinium oceanicum

++
++

$++$

$++$

$+++\quad++$

$++$

(VanHöffen, 1897) Balech 1974 
Efecto de las descargas del emisario submarino de aguas residuales de la ciudad de Limón sobre la calidad del agua, abundancia y diversidad del fitoplancton en los alrededores de isla Uvita, Costa Rica

\section{Estación}

\section{Especie}

Protoperidinium pellucidum Bergh 1881

Protoperidinium pentagonum

(Gran) Balech 1974

Protoperidinium sp.

Pyrophacus steinii (Schiller)

Wall \& Dale 1971

Scrippsiella trochoidea (F.Stein)

A. R.Loeblich III 1976

Dinoflagelado no id

\section{Cyanophyceae}

Ciano no id 1

Oscillatoria sp.

Phormidium sp.

Synechocystis sp.

Johannesbaptistia pellucida

(Dickie) W.R.Taylor \& Drouet in

Drouet 1938

\section{Chlorophyceae}

Euglena sp.

Eutreptiella sp.

\section{Otros grupos}

Fitoflagelado no id

E-1

E-2 +

$\begin{array}{ll}\mathbf{E}-3 & \mathbf{E}-4 \\ + & +\end{array}$

E-4
++
+
++
+

$+$

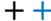

E-5 E-6

$++$

$+\quad+$

$\begin{array}{llll}+ & + & + & + \\ + & & + \\ & & +\end{array}$

$+\quad+$

$+$

+
+
+

\section{$+$}

$+\quad+$

$+\quad+$

$+\quad+$

$++\quad++$

$\begin{array}{llllllllllllllllll}+ & + & + & + & + & + & +\end{array}$

$+$

$+\quad+$

+ Muestreo 10/09/2012. + Muestreo 11/10/2012. +

A 10 metros de profundidad y en la región de influencia del emisario, se obtuvieron índices de diversidad bajos en comparación con su contraparte en la superficie; mientras que en la región fuera de la influencia se registraron valores levemente mayores, que oscilan entre 2.44 bits/cél y 2.85 bits/cél. La dominancia fue baja en todos los puntos de muestreo, con un mínimo de 0.06 bits/cél y el máximo de 0.23 bits/cél.

Según el diagrama de OlmsteadTukey (Fig. 5) 34 especies fueron dominantes, una de las cuales fue el dinoflagelado Protoperidinium sp. y el resto diatomeas. De estas, T. nitzschioides y $S$. costatum 

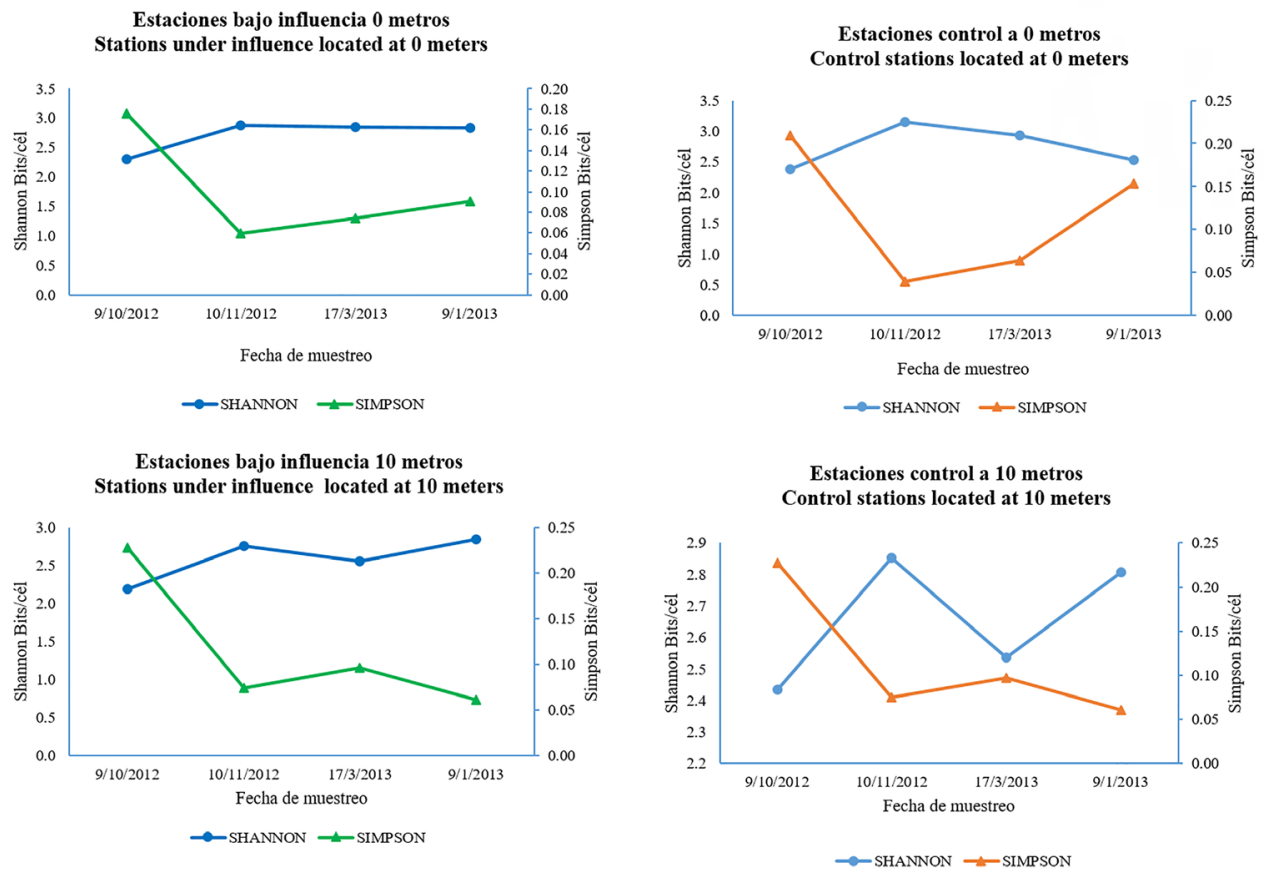

Fig. 4. Valor promedio de los Índices de diversidad Shannon Wiener y dominancia de Simpson para estaciones bajo influencia del emisario submarino y sus controles según profundidad

Fig. 4. Average value of the Shannon Wiener diversity index and Simpson's dominance index for stations under the influence of the marine outfall and its controls according to depth

fueron las más frecuentes, seguidas por Pseudonitzschia pungens, junto con Chaetoceros descipiens y $R$. stolterfothii. Las especies consideradas como raras fueron 29, entre ellas 2 cianobacterias, 12 dinoflagelados y 19 diatomeas. Entre los dinoflagelados más frecuentes estuvieron Prorocentrum micans y Prorocentrum minimum, seguidos por Protoperidinium oceanicum y Protoperidinium pellucidum. Las especies raras más frecuentes de diatomeas fueron Rhizosolenia hebetata, Rhizosolenia acuminata junto con Chaetoceros atlanticus. Las especies ocasionales fueron 9; entre ellas 3 dinoflagelados y 6 diatomeas, de las cuales Chaetoceros didymus y Chaetoceros compressus fueron los más frecuentes. Para esta investigación no se registraron especies constantes.

Con respecto a la abundancia de microalgas se logró evidenciar que no existe diferencia significativa entre la zona de influencia y los controles $(R=$ $0.069 P=0.12)$, pero sí entre la superficie y el fondo $(R=0.14 P=0.001)$. 
Efecto de las descargas del emisario submarino de aguas residuales de la ciudad de Limón sobre la calidad del agua, abundancia y diversidad del fitoplancton en los alrededores de isla Uvita, Costa Rica

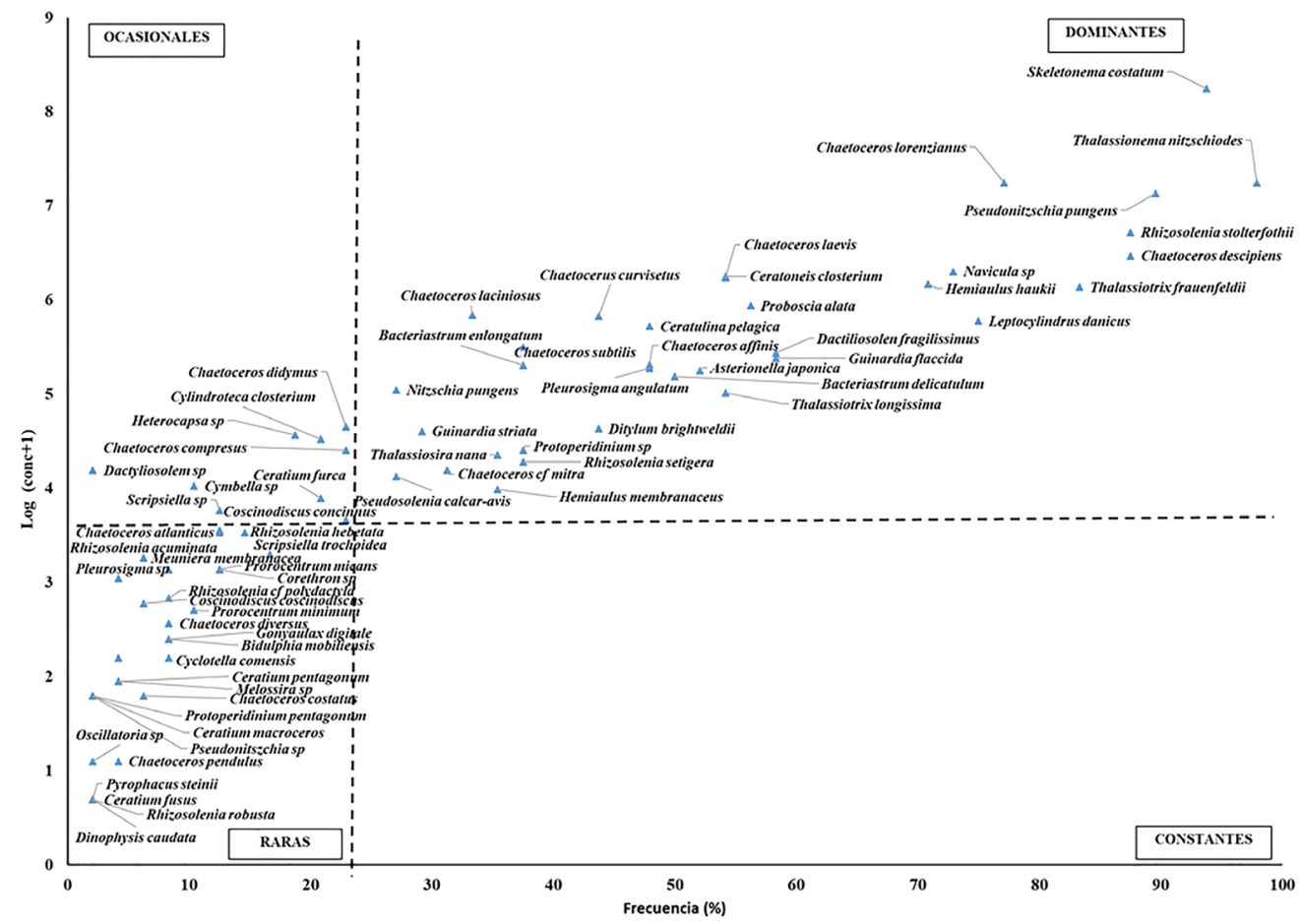

Fig. 5. Diagrama de Olmstead- Tukey de las especies fitoplanctónicas para 6 estaciones de muestreo alrededor de isla Uvita; Limón, Costa Rica

Fig. 5. Olmstead-Tukey diagram of phytoplankton species for six sampling stations around Uvita Island; Limón, Costa Rica

En total, 22 especies fitoplanctónicas fueron las que aportaron las mayores disimilitudes entre la superficie $y$ el fondo. S. costatum fue una de las más importantes, con una concentración promedio en la superficie de 2000 cél/L y de 1350 cél/L en el fondo, lo que corresponde a un aporte del 18.5\% de disimilitud. Otras especies como Cerataulina pelagica, Hemiaulus hauckii y Chaetoceros curvisetus aportan valores de disimilitud de entre 3.0 y 2.2 (Cuadro 3 ).
Un total de 33 especies fitoplanctónicas y 10 variables ambientales fueron utilizadas para el análisis de correspondencia canónica (Fig. 6). La respuesta de las especies a las variables ambientales fue explicada, en específico, por los tres primeros ejes $(71 \%)$, siendo el primero el más importante con un $33.3 \%$. Este eje se halla fuertemente relacionado con la precipitación, la temperatura, el amonio, y los silicatos. Por otro lado, las variables temperatura, silicato, nitrito y oxígeno disuelto son las más destacadas para el segundo eje. 
Cuadro 3. Análisis SIMPER de las disimilitudes promedio en la concentración de especies fitoplanctónicas entre estaciones bajo influencia del emisario submarino y sus controles

Table 3. SIMPER analysis of the average dissimilarities of phytoplankton concentration between stations under the influence of the marine outfall and its controls

\begin{tabular}{|c|c|c|c|c|}
\hline Especie & $\begin{array}{l}\text { Promedio } \\
\text { Superficie } \\
\text { (cél/L) }\end{array}$ & $\begin{array}{l}\text { Promedio } \\
\text { fondo } \\
\text { (cél/L) }\end{array}$ & $\begin{array}{l}\text { Sd de la } \\
\text { disimilitud }\end{array}$ & $\begin{array}{l}\text { \% de } \\
\text { contribución }\end{array}$ \\
\hline Skeletonema costatum & 2000 & 1350 & 1.1 & 18.5 \\
\hline Pseudonitzschia pungens & 1083 & 398 & 0.7 & 9.4 \\
\hline Chaetoceros lorenzianus & 840 & 435 & 1.3 & 7.4 \\
\hline Thalassionema nitzschioides & 835 & 833 & 1.2 & 6.3 \\
\hline Rhizosolenia stolterfothii & 750 & 292 & 1.1 & 5.3 \\
\hline Asterionella japonica & 354 & 221 & 0.5 & 3.7 \\
\hline Nitzschia pungens & 260 & 144 & 0.6 & 3.6 \\
\hline Chaetoceros decipiens & 473 & 191 & 1.2 & 3.5 \\
\hline Navicula sp. & 179 & 344 & 0.6 & 3.4 \\
\hline Cerataulina pelagica & 314 & 113 & 1.0 & 3.0 \\
\hline Leptocylindrus danicus & 300 & 190 & 1.2 & 2.6 \\
\hline Dactyliosolen fragilissimus & 271 & 65 & 0.9 & 2.5 \\
\hline Hemiaulus hauckii & 317 & 77 & 1.0 & 2.4 \\
\hline Thalassiotrix frauenfeldii & 300 & 223 & 1.2 & 2.3 \\
\hline Chaetoceros laevis & 235 & 79 & 0.8 & 2.3 \\
\hline Chaetoceros curvisetus & 288 & 25 & 0.8 & 2.2 \\
\hline Ceratoneis closterium & 154 & 135 & 1.1 & 2.0 \\
\hline Chaetoceros subtilis & 133 & 54 & 0.8 & 1.6 \\
\hline Thalassiothrix longissima & 146 & 56 & 0.9 & 1.4 \\
\hline Ditylum brighthwellii & 119 & 88 & 0.8 & 1.4 \\
\hline Bacteriastrum delicatulum & 119 & 67 & 1.0 & 1.4 \\
\hline Chaetoceros laciniosus & 152 & 27 & 0.5 & 1.3 \\
\hline Chaetoceros affinis & 119 & 63 & 1.0 & 1.3 \\
\hline Proboscia alata & 108 & 96 & 1.1 & 1.2 \\
\hline
\end{tabular}


Efecto de las descargas del emisario submarino de aguas residuales de la ciudad de Limón sobre la calidad del agua, abundancia y diversidad del fitoplancton en los alrededores de isla Uvita, Costa Rica

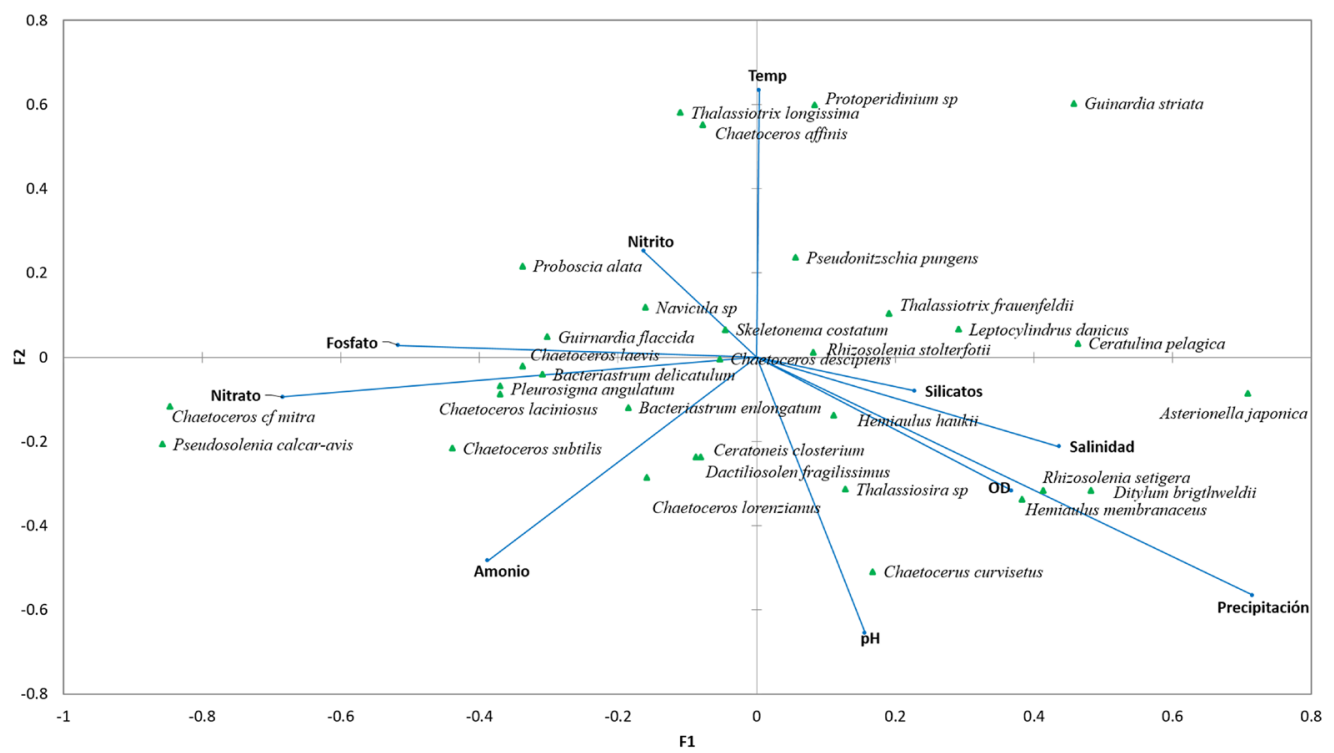

Fig. 6. Proyección del análisis de correspondencia canónica del fitoplancton y las variables biológicas para las estaciones bajo influencia del emisario submarino y sus controles

Fig. 6. Projection of the canonical correspondence analysis of phytoplankton and biological variables for stations under the influence of the marine outfall and its controls

En el primer cuadrante del biplot y relacionadas con la variación en la precipitación y la temperatura, se encontraron las especies Guinardia striata, P. pungens, Thalassionema frauenfeldii. La variación de la salinidad fue de particular importancia para las diatomeas C. pelagica, L. danicus y Asterionella japonica que se ubican hacia los extremos del primer eje, mientras que el aumento en la precipitación está correlacionado con el aumento en la concentración de Rhizosolenia setigera, Ditylum brightwellii y Hemiaulus membranaceus. En el tercer $\mathrm{y}$ cuarto cuadrante se identificaron especies agrupadas por los nutrimentos nitrato, ortofosfato y amonio, donde Chaetoceros laciniosus, Chaetoceros laevis y Ch. descipiens estuvieron más relacionados con la concentración de ortofosfato, mientrasquePseudosolenia calcar-avis y Chaetoceros cf. mitra se ubican en los extremos del eje nitrato. Por otro lado, el $\mathrm{pH}$ y la concentración de amonio explican la abundancia de Cylindrotheca closterium, Dactyliosolen fragilissimus y Chaetoceros lorenzianus, que se relaciona con los valores bajos de amonio. 


\section{DISCUSIÓN}

En algunas temáticas el Caribe costarricense continúa siendo inexplorado, los estudios publicados sobre la diversidad y abundancia de microalgas fitoplanctónicas y su relación con los parámetros fisicoquímicos son prácticamente inexistentes, a la fecha solo existe un estudio de impacto ambiental previo a la construcción del emisario (PROIGEAyA, 1999) y el realizado por MoraAlvarado \& Portuguez-Barquero (2016), pero respecto a la abundancia y diversidad algal no existe referente para un "antes "o un "después" en la zona, por ello este estudio es pionero y su información es sumamente valiosa para futuras investigaciones en la temática.

El análisis de componentes principales y de correspondencia canónica evidenciaron que en la zona de muestreo existe influencia de las precipitaciones, donde los valores altos de silicatos, oxígeno disuelto y sólidos totales suspendidos coinciden con las mayores precipitaciones. Troccoli et al. (2004), obtienen patrones similares en la península de Yucatán, en una zona con influencia de agua dulce; sin embargo, a diferencia de estos autores, durante los períodos de lluvias no se encontraron especies de microalgas típicas de ecosistemas dulceacuícolas.

Los datos reflejan que hay influencia de las precipitaciones sobre los factores abióticos y, por ende, en la estructura de la comunidad fitoplanctónica. Durante los períodos de mayores precipitaciones, que corresponden al primer y tercer muestreo, se observó un aumento en la concentración de silicato, nitrato, amonio y fosfato, lo que sugiere que la zona de estudio se encuentra bajo la influencia del caudal de los ríos en la región, tal y como lo mencionaron Brenes-Rodríguez \& Benavides-Morera (2015).

La alta escorrentía desde la zona terrestre junto con el arrastre de sedimentos y el aumento en la concentración de nutrimentos podría ser también responsable del aumento de los sólidos totales durante el período, a través de dos mecanismos: 1) partículas en suspensión derivadas de los procesos de erosión, meteorización y arrastres de materia orgánica (Vinayachandran et al. 2002; Vajravelu et al. 2018) y 2) aumento de la biomasa microalgal estimulada por el ingreso de nuevos nutrientes (Quinlan \& Philips, 2007).

La diferencia entre el fondo y la superficie respecto a los parámetros físico-químicos, puede ser producto de la naturaleza misma de la columna de agua y no de la operación del emisario submarino. Estas diferencias en parámetros tales como el oxígeno, tienden a aumentar conforme aumenta la profundidad, producto de factores como la disminución de la cantidad de organismos fotoautótrofos y a una relación más cercana con el fondo, donde se pueden presentar procesos 
Efecto de las descargas del emisario submarino de aguas residuales de la ciudad de Limón sobre la calidad del agua, abundancia y diversidad del fitoplancton en los alrededores de isla Uvita, Costa Rica

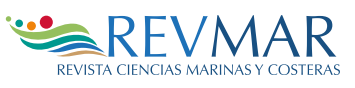

de descomposición y reciclaje de nutrientes. Los valores obtenidos fueron similares a los reportados por el PROIGE- AyA (1999), previo a la construcción del emisario, por lo que se puede asegurar que no existe una afectación en el área de estudio respecto a los parámetros físico-químicos.

$$
\text { Mora-Alvarado y Portu- }
$$
guez-Barquero en el 2016, reportaron 33 UFC de Enterococcus para julio del 2014, una cantidad mayor a la reportada en el presente estudio, tanto en las estaciones con influencia del emisario como en los controles. Para dictaminar si un cuerpo de agua es apto para la recreación se utiliza como referencia la normativa estadounidense, que establece un máximo de $35 \mathrm{UFC} / 100 \mathrm{ml}$ (USEPA, 2012; Vergaray et al. 2007). En el nivel latinoamericano se pueden tener como referentes a México (COFEPRIS, 2020) con un límite máximo de $200 \mathrm{UFC} / 100 \mathrm{~mL}$ o su equivalente de $200 \mathrm{NMP} / 100 \mathrm{~mL}$ y Cuba (Miravet et al. 2009) con un máximo permisible $\leq 100 \mathrm{NMP} / 100 \mathrm{~mL}$. Bajo estas normativas las aguas en los puntos de muestreo, de esta investigación, podrían clasificarse como aptas para el uso recreativo.

Los controles positivos permitieron verificar la no afectación de las descargas del río Cieneguita sobre la cantidad de Enterococcus en las estaciones ubicadas al sur de isla Uvita. La carga del río fue hasta 50 veces mayor que la establecida por la USEPA (2012), como mínima para proteger la salud humana. Estas descargas podrían estar canalizándose hacia la corriente superficial que se mueve paralela a la playa, por lo que es recomendable tomar muestras en esos puntos, que son, justamente, los utilizados por los bañistas y pescadores para realizar sus actividades.

No hubo diferencia significativa en la cantidad de UFC entre la zona bajo influencia del emisario submarino y sus controles, por ello no se puede asumir una afectación al medio causada por los efluentes vertidos. Los controles positivos mostraron valores muy altos en comparación con las estaciones de muestreo; esto podría indicar que el río Cieneguita es un punto muy importante de contaminación fecal y que su problemática debería abordarse desde una perspectiva interdisciplinaria.

En otras latitudes, García y Luque (2011) reportan concentraciones algales máximas que rondan las 4000 cél/L alrededor de las islas Canarias, un entorno con influencia oceánica y escases de nutrientes, mientras que en la bahía Boka Kotorska, Drakulović et al. (2016) reportan valores máximos de $8.4 \times 10^{5}$ cél $/ \mathrm{L}$, en un ecosistema fuertemente influenciado por la actividad antropogénica. La densidad algal reportada en los alrededores del emisario submarino se encuentra apenas por encima de los valores de un sistema de características oligotróficas (García \& Luque, 2011). 
Las diatomeas dominaron en todas las muestras seguidas por los dinoflagelados, y este comportamiento también ha sido observado en otras regiones del mundo (García \& Luque, 2011; Muhammad-Adlan et al. 2012; Drakulović et al. 2016). Los datos obtenidos por Hulburt (1968), cerca de la costa Caribe costarricense, donde se logró identificar un total de 15 especies, es un número bajo en comparación con el encontrado en esta investigación.

La diversidad algal en las estaciones de muestreo tendió a ser alta mientras que la dominancia fue baja; este comportamiento coincide con lo reportado por García y Luque (2011), para 15 estaciones de muestreo ubicadas en la costa del municipio de San Bartolomé de Tirajana en islas Canarias. Margalef (1986), indica que el índice de diversidad en ecosistemas costeros puede variar entre 3.5 y 4.5 , dado que los valores de diversidad obtenidos en la zona se mantuvieron en 3 , la comunidad fitoplanctónica podría ser considerada dominantemente costera.

La zona de estudio es un ambiente del tipo costero, donde al parecer la abundancia de las especies y la secuencia en la que se presentan puede no implicar un proceso de sucesión, sino más bien una serie de eventos temporales, causados por fenómenos físicos como corrientes, el tiempo o escorrentías. Lo anterior se refleja por algunas de las especies como T. nitzschioides, y S. costatum, que dominan ecosistemas costeros designados por Margalef (1997) como "turbulentos", donde las aguas están mezcladas y cargadas de nutrientes. La alta relación superficie-volumen de estas diatomeas les confiere una rápida capacidad de crecimiento y absorción de nutrientes por lo que pueden alcanzar altas densidades en poco tiempo.

El 57\% de las especies fitoplanctónicas fueron raras, esto debido a que presentaron densidades y frecuencias de aparición inferiores a la media. Lo anterior, sumado a la ausencia de especies constantes, podría deberse a un cambio continuo en la dinámica del ecosistema, donde las poblaciones algales son removidas de la zona por la corriente marina sin que la tasa de división celular pueda reponer las células perdidas; lo que concuerda con los resultados obtenidos por Varona-Cordero \& Gutiérrez-Mendieta (2003) y por Varona-Cordero et al. (2010), en lagunas costeras de México.

Las diferencias observadas entre la abundancia algal de la superficie y del fondo reflejan el comportamiento típico del fitoplancton marino y no una afectación del emisario submarino. De las dos especies que dominaron a 10 $\mathrm{m}$ de profundidad, Navicula $\mathrm{sp}$. es dominantemente bentónica, por lo que es usual encontrarla a esta profundidad. Mientras que, R. stolterfothii, mostró colonias pequeñas, que según lo reportado por Peperzak et al. (2003), podrían presentar tasas de asentamiento 
Efecto de las descargas del emisario submarino de aguas residuales de la ciudad de Limón sobre la calidad del agua, abundancia y diversidad del fitoplancton en los alrededores de isla Uvita, Costa Rica

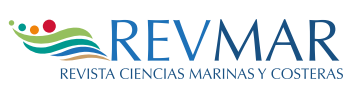

mayores. El alto porcentaje de disimilitud aportado por S. costatum y $C h$. lorenzianus puede deberse al mayor tamaño de las colonias, factor que contribuye a que presente flotabilidad neutra (Cloern et al. 1983), al igual que $P$. pungens forman cadenas de variada longitud y alargadas, que facilita su reubicación en las capas superficiales, por los movimientos de las masas de agua.

Muchas de las especies presentes en la zona son cosmopolitas y presentan múltiples mecanismos para adaptarse a entornos dinámicos. Sin embargo, a pesar de esto requieren de condiciones óptimas para mantener la densidad poblacional. Khan et al. (1998) y Tian et al. (2002) demostraron, bajo condiciones de laboratorio, que $S$. costatum crece en rangos de salinidad de 25-35 PSU y temperaturas de $20-25^{\circ} \mathrm{C}$, circunstancias similares a las observadas en el presente estudio. Esto podría explicar las altas densidades y la dominancia de la especie a lo largo del estudio.

S. costatum se encuentra ampliamente distribuida en los mares del mundo y produce florecimientos nocivos bajo condiciones eutróficas, por lo que se ha sugerido como indicador de la calidad del agua (Sánchez et al. 1995; Jaanus et al. 2009). Sin embargo, a pesar de que la especie es dominante y aporta la mayor densidad de células, no existe en el área de estudio evidencia documentada de florecimientos algales y, más bien, existe una dinámica similar a la reportada por Vilicic et al. (1995).

La temperatura fue el factor más importante con el que se relacionó la abundancia de $P$. pungens y concuerda con lo reportado por Zrinka et al. (2011), para el noreste del mar Adriático. La especie es cosmopolita y se ha relacionado su abundancia con el ortofosfato y el amonio (Zrinka et al. 2011; Trainer et al. 2012). No obstante, el presente estudio muestra que puede existir especificidad regional y que otros factores pueden explicar la abundancia de la especie.

Estudios en otras latitudes han evidenciado que un aumento en la ocurrencia de Chaetoceros sp. y Bacteriastrum sp. puede estar asociada con altas concentraciones de nutrientes (Kumar et al. 2007; Paul et al. 2008). Pese a que esta información pareciera reflejarse en el análisis de correspondencia canónica, es importante recalcar que los valores de nutrimentos, en general, se mantienen bajos con respecto a zonas con mayor productividad y, por ello, no debe interpretarse esta relación como un mero indicador de eutrofización antropogénica, sino más bien desde la perspectiva de la eficiencia y especificidad en el uso del nitrógeno y el fósforo.

Los datos recolectados en el presente estudio han demostrado que no existe un impacto evidente en el medio marino adyacente a la zona de 
desfogue del emisario submarino de la ciudad de Limón, y que la estructura y composición de la comunidad fitoplanctónica, dominada por diatomeas céntricas se encuentra muy influenciada por la precipitación, la temperatura, el amonio y los silicatos.

\section{REFERENCIAS}

Ávalos, A. (2004). Limón ya tiene un sistema de aguas negras. La Nación. https:// www.nacion.com/el-pais/limon-ya-tiene-un-sistema-de-aguas-negras/HGXD26REZVHRZBO4OCX7O6ZATI/ story/

Brenes-Rodríguez, C. L. \& Benavides-Morera, R. (2015). Características termohalinas de las aguas costeras del Caribe Sur de Costa Rica. Rev. Mar. Cost., 7, 27-41. http://dx.doi.org/10.15359/ revmar.7.2.

Carvajal, M. (2005). Sistema de aguas negras de Limón Opera al 100 por ciento. $L a$ Nación. https://www.nacion.com/elpais/sistema-de-aguas-negras-de-limon-opera-al-100-por-ciento/FOODNXQRCFAKNHIPRJ65ICPNXU/ story/

Chinchilla, R. G. (2013). Resumen meteorológico setiembre de 2013. Costa Rica. Instituto Metereológico Nacional.

Clesceri, L., Greenberg, A. \& Eaton. A (Eds.) (1999). Standard methods for the examination of water and wastewater. EE.UU.: American Public Health Association

Cloern, J. E., Alpine, A. E., Cole, B. E., Wong, J. F. \& Ball M.D. (1983). River discharge controls phytoplankton dynamics in the northern San Francisco Bay Estuary. Estuar. Coast.
Nelson Muñoz Simon, Lilliana Piedra Castro, Ricardo Jiménez Montealegre, José Pereira Chaves, Meyer Guevara Mora y Gilberto Piedra Marín

Shelf Sci., 16(4), 415-429. https://doi. org/10.1016/0272-7714(83)90103-8

COFEPRIS. (2020). Manual operativo: monitoreo de agua de contacto primario en el agua de mar de playas y cuerpos de agua dulce. https:/www.gob.mx/ cofepris/documentos/manual-operativo-monitoreo-de-agua-de-contactoprimario-en-el-agua-de-mar-de-playas-y-cuerpos-de-agua-dulce

Cuello, V. G., Llanos, E. N., Garaffo, G. V. \& Jaubet, M. L. (2019). Emisario submarino de Mar del Plata (Argentina): ¿Cómo impactó su construcción en la comunidad bentónica intermareal? Ecol. Austral., 29, 028-040. https://doi. org/10.25260/EA.19.29.1.0.771

Drakulović D., Pestorić B., Kraus R., Ljubimir S. \& Krivokapić S. (2016). Phytoplankton Community and Trophic State in Boka Kotorska Bay. In A. Joksimović, M. Djurović, A. Semenov, I. Zonn \& A. Kostianoy (Eds.), The Boka Kotorska Bay Environment (pp 169-201). Switzerland: Springer.

García, R. A. \& Luque, E. A. (2011). Evaluación ambiental de las aguas costeras y puertos deportivos a partir de la comunidad fitoplanctónica en las Islas $\mathrm{Ca}$ narias, España. Tesis de doctorado no publicada, Universidad de las Palmas de Gran Canarias, Gran Canarias.

Gianesella, F. M., Saldanha-Corrêa, P, M., Kutner, B. M. \& Pompeu, M (1999). Assessment of plankton community and environmental conditions in São Sebastião Channel prior to the construction of a produced water outfall. Rev. Brasileira Oceanogr., 47(1), 29-46. https://doi.org/10.1590/ S1413-77391999000100003.

Hansen, H. \& Koroleff, F. (2007). Determination of nutrients. In K. Grasshoff \& M. Ehrhardt (Eds.), Methods of Seawater Analysis (pp. 159-228). 
Efecto de las descargas del emisario submarino de aguas residuales de la ciudad de Limón sobre la calidad del agua, abundancia y diversidad del fitoplancton en los alrededores de isla Uvita, Costa Rica

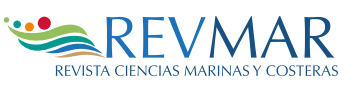

EE. UU.: Wiley-VCH. https://doi. org/10.1002/9783527613984.ch10

Hulburt, E. M (1968). Phytoplankton Observations in the Western Caribbean Sea. Bull. Mar. Sci.,18(2), 388-399.

Instituto Meteorológico Nacional. (2016). Descripción del clima cantón de Limón. http://cglobal.imn.ac.cr/ documentos/publicaciones/DescripciondelClimaSERIE/DescripcionClimaCantonLimon/html5/index. html?page $=1 \&$ noflash

Jaanus, A., Toming, K., Hällfors, S. Kaljurand, K \& Lips, I. (2009). Potential phytoplankton indicator species for monitoring Baltic coastal waters in the summer period. Hydrobiologia, 629, 157-168. https://doi. org/10.1007/s10750-009-9768-y

Khan, S., Haque, M., Arakawa, O. \& Onoue, Y. (1998). Physiological observations on a diatom Skeletonema costatum (Greville) Cleve. Bangladesh J. Fish. Res., 2, 109-118.

Kumar, S. S., Nuncio, M. S., Ramaiah, N., Sardesai, S., Narvekar, J., Fernandes, V. \& Paul, J. T. (2007). Eddy-mediated biological productivity in the Bay of Bengal during fall and spring intermonsoons. Deep-Sea Res., 9(54), 1619-1640. https://doi.org/10.1016/j. dsr.2007.06.002

Margalef, R. (1997). Turbulence and marine life. Sci. Mar., 61(1), 109-123.

Marín, R., Marquetti, M. \& Díaz, M. (2009). Índices larvales de Aedes aegypti antes y después de intervenciones de control en Limón, Costa Rica. Rev. Cub. de Med. Trop., 2(61),1-10.

Miravet, M. E, Lugioyo, M., Loza, S., Enrique, D., Delgado, Y., Carmenate, M. \& Pérez, D. M. (Eds.). (2009). Procedimientos para el monitoreo de la calidad ambiental en la zona marino costera a partir de microorganismos. Cuba. Ediciones Centenario

Mora-Alvarado, D. \& Portuguez-Barquero, C. (2016). Cobertura de la disposición de excretas en Costa Rica en el periodo 2000-2014 y expectativas para el 2021. Rev. Tec. Marcha, 29(2), 43-62. https:// doi.org/10.18845/tm.v29i2.2690

Morera, R. R. (2012). Resumen meteorológico setiembre de 2012. https://www. imn.ac.cr/documents/10179/14635/ SEPTIEMBRE.

Muhammad-Adlan, A. H., Wan-Maznah, W.O., Khairun, Y., Chuah, C. C., Shahril, M. H. \& Noh, M. (2012). Tropical Marine Phytoplankton Assemblages and Water Quality Characteristics Associated with Thermal Discharge from a Coastal Power Station. J. Nat. Sci. Res., 2, 88-99.

Naranjo, D. J. (2013). Resumen meteorológico marzo de 2013. https://www.imn.ac.cr/ documents/10179/14637/MARZO.

Naranjo, D. J. \& Poleo, B. D. (2012). Resumen meteorológico octubre de 2012. https://www.imn.ac.cr/ documents/10179/14635/OCTUBRE.

Oksanen, J., Blanchet, F. G., Kindt, R., Legendre, P., Minchin, P. R. ... \& Wagner, H. (2019). Ordination methods, diversity analysis and other functions for community and vegetation ecologists. Vegan: Community Ecology Package. $R$ Package Version 2.5-7. Austria: R Foundation for Statistical Computing.

Parnell, P. E. (2003). The effects of sewage discharge on water quality and phytoplankton of Hawai'ian coastal waters. Mar. Env. Res., 55(4), 293-311. https://doi.org/ 10.1016/ S0141-1136(02)00275-1

Paul, J. T., Ramaiah, N. \& Sardessai, S. (2008). Nutrient regimes and their effect on distribution of phytoplankton in the Bay of Bengal. Mar. Envir. Res., 
66, 337-344. https://doi.org/10.1016/j. marenvres.2008.05.007.

Peperzak, L., Colijn, F., Koeman, R., Gieskes, W. W. \& Joordens, J. C. (2003). Phytoplankton sinking rate in the Rhine region of freshwater influence. J. Plankton Res., 25(4), 365-383. https://doi. org/10.1093/plankt/25.4.365

PROIGE-AyA. (1999). Informe. Estudio de impacto ambiental del proyecto emisario submarino para la Ciudad de Limón. Costa Rica: Sin editorial.

Quinlan, E. L. \& Philips, E. J. (2007). Phytoplankton assemblages across the marine to low-salinity transition zone in a blackwater dominated estuary. $J$. Plankton Res., 29(5), 401-416. https:// doi.org/10.1093/plankt/fbm024

Ramírez, C. J. \& Salazar, M. E. (2005). Calidad de aguas residuales en los sistemas de depuración operados y administrados por AYA y estudios especiales de interés institucional (Informe 2005). Costa Rica: Sin editorial.

Salas, H. J. (2000). Emisarios submarinos alternativa viable para la disposición de aguas negras de ciudades costeras en América Latina y el Caribe. Perú: CEPIS.

Sánchez, S., Martínez, M. E., Molina, E. \& Casa, J. A. (1995). Skeletonema costatum as a potential source of fatty acids and single-cell protein (SCP): The effect of $\mathrm{pH}$ on growth rate and biomass composition. Mar. Biotechnol., 2, 23-26.

Sokal, R. R. \& Rohlf, F. J. (1970). Biometry: the principles and practice of statistics in biological research. Journal of the Royal Statistical Society. Series A (General), 133(1), 102-102. https://doi. org/10.2307/2343822

Sournia, A. (1978). Phytoplankton Manual. France: UNESCO
Nelson Muñoz Simon, Lilliana Piedra Castro, Ricardo Jiménez Montealegre, José Pereira Chaves, Meyer Guevara Mora y Gilberto Piedra Marín

Stanca, E. y Parsons, L. (2017). Phytoplankton diversity along spatial and temporal gradients in the Florida Keys. J. Plankton Res., 39(3), 531-549. https://doi. org/10.1016/S0141-1136(02)00275-1

Tian, Y., Mingjiang, Z. \& Peiyuan, Q. (2002). Combined effects of temperature, irradiance and salinity on growth of diatom Skeletonema costatum. Chin. J. Ocean. Limnol., 20, 237-243. https:// doi.org/10.1007/BF02848852

Tinoco, Q. J. (2007). Manual de técnicas analíticas para la determinación de parámetros fisicoquímicos y contaminantes marinos: Aguas, sedimentos y organismos. Colombia: INVEMAR.

Toledo, H., Hernández, C., Rodríguez, C., Bittner, V., Ferreira, L. \& Orellana, F. (2005). Estudio de la contaminación fecal mensual y estacional en la zona costera adyacente al emisario submarino en la bahía de puerto Montt. Gayana (Concepc.), 69(1), 104-112. http://dx.doi.org/10.4067/ S0717-65382005000100011

Trainer, V. L, Stephen, S. B., Lundholm, N., Thessen, A. E., Cochlan, P. W., Adams, N. G. \& Trick, G. C. (2012). Pseudo-nitzschia physiological ecology, phylogeny, toxicity, monitoring and impacts on ecosystem health. Harmful Algae, 14(2012), 271-300. https://doi. org/10.1016/j.hal.2011.10.025

Troccoli, G. L., Herrera-Silveira, J. \& Comín, F. A. (2004). Structural variations of phytoplankton in the coastal seas of Yucatan, Mexico. Hydrobiologia, 519, 85-102. https://doi.org/10.1023/B:HYDR.0000026487.78497.b6

USEPA. (2006). Method 1600: Enterococci in Water by Membrane Filtration Using membrane-Enterococcus Indoxyl- $\beta-D$-Glucoside Agar ( $m E I$ ). (Report \# 821-R-02-022). EE. UU.: Government printing office. 
Efecto de las descargas del emisario submarino de aguas residuales de la ciudad de Limón sobre la calidad del agua, abundancia y diversidad del fitoplancton en los alrededores de isla Uvita, Costa Rica

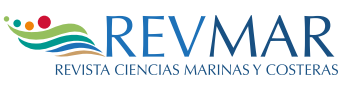

USEPA. (2012). Recreational Water Quality Criteria. https://www.epa.gov/wqc/2012-recreational-water-quality-criteria

Vajravelu, M., Mariasingarayan, Y., Saravanakumar, A. \& Mayakrishnan, M. (2018). Seasonal influence of physico-chemical parameters on phytoplankton diversity, community structure and abundance at Parangipettai coastal waters, Bay of Bengal, South East Coast of India. Oceanologia, 60(2), 114-127. https://doi.org/10.1016/j. oceano.2017.08.003

Varona-Cordero, F. \& Gutiérrez-Mendieta, F. (2003). Estudio multivariado de la fluctuación espacio-temporal de la comunidad fitoplanctónica en dos lagunas costeras del estado de Chiapas. Hidrobiológica, 13(3), 177-194.

Varona-Cordero, F., Gutiérrez-Mendieta, F. \& Castillo, M. M. (2010). Phytoplankton assemblages in two compartmentalized coastal tropical lagoons (Carretas-Pereyra and Chantuto-Panzacola, Mexico). J. Plankton Res., 32, 1283-1299. https://doi.org/10.1093/plankt/fbq043

Vergaray, G., Méndez, C.R., Morante, H. Y., Heredia, V. \& Béjar, V. (2007). Enterococcus y Escherichia coli como indicadores de contaminación fecal en playas costeras de Lima. Rev. Inst. Inves. FIG$M M G$., 10(20), 82-86.

Vidal, T., Calado, A., Moita, M., \& Cunha, M. (2017). Phytoplankton dynamics in relation to seasonal variability and upwelling and relaxation patterns at the mouth of Ria de Aveiro (West Iberian Margin) over a four-year period. $\mathrm{PloS}$ one, 12(5), e0177237. https://doi. org/10.1371/journal.pone.0177237

Vilicic, D., Krsinic, F., Caric, M., Jasprica, N., Bobanovic, S. \& Mikus, J. (1995). Plankton and hydrography in a moderately eutrophicated eastern Adriatic bay
(Gruz Bay). Hydrobiologia, 304, 9-22. https://doi.org/10.1007/BF00006876

Vinayachandran, P. N., Murty, V. S. \& Ramesh Babu, V. (2002). Observations of barrier layer formation in the Bay of Bengal. J. Geophys. Res. 107 (12), 1-14. https://doi.org/10.1029/2001JC000831

Zrinka, L., Suncica, B., Damir, V., Borojevic, K., Pfannkuchen, D., Jelena, G., Ivana, U., Peharec Štefanić, P. \& Djakovac, T. (2011). Ecology and taxonomy of potentially toxic Pseudo-nitzschia species in Lim Bay (north-eastern Adriatic Sea). Harmful Algae, 10, 713-722. https:// doi.org/10.1016/j.hal.2011.06.002 
